Published in final edited form as:

Antivir Ther. 2017 ; 22(8): 669-680. doi:10.3851/IMP3157.

\title{
Neurocognitive Dysfunction in HIV-Infected Youth: Investigating the Relationship with Immune Activation
}

\author{
Allison Ross Eckard, MD ${ }^{1,2}$, Julia C. Rosebush, DO ${ }^{1}$, Mary Ann O'Riordan, PhD $^{3}$, Chanda \\ C. Graves, PhD ${ }^{1}$, Ashley Alexander, PsyD ${ }^{4}$, Anita K. Grover, PsyD ${ }^{5}$, S. Thera Lee, BS ${ }^{1}$, \\ Jakob G. Habib, BS ${ }^{1}$, Joshua H. Ruff, BS ${ }^{1}$, Ann Chahroudi, MD, PhD ${ }^{1,4}$, and Grace A. \\ McComsey, $\mathrm{MD}^{3}$ \\ ${ }^{1}$ Emory University School of Medicine, Atlanta, GA, USA \\ ${ }^{2}$ Medical University of South Carolina, Charleston, SC, USA \\ ${ }^{3}$ Rainbow Babies \& Children's Hospital and Case Western Reserve University School of \\ Medicine, Cleveland, $\mathrm{OH}$, USA \\ ${ }^{4}$ Children's Healthcare of Atlanta, Atlanta, GA, USA \\ ${ }^{5}$ Georgia State University, Atlanta, GA, USA
}

\section{Abstract}

Background-HIV-infected individuals are at increased risk of neurocognitive impairment compared to the general population. Studies suggest that, despite combination antiretroviral therapy (cART), HIV infection causes immune activation which results in neural damage; however, few data exist in HIV-infected youth.

\begin{abstract}
Methods-HIV-infected youth 8-26 years old on cART with virologic suppression were prospectively enrolled along with healthy controls. Neurocognitive performance was assessed by age-appropriate Wechsler Intelligence Scales. Soluble and cellular markers of T-lymphocyte and monocyte activation were measured by ELISA and flow cytometry, respectively.

Results-45 HIV-infected subjects and 21 controls were enrolled. Markers of T-cell and monocyte activation were higher in the HIV-infected subjects compared to controls, but proportions of inflammatory and patrolling monocytes were similar. Although there was no significant differences in neurocognitive scores between the HIV-infected and control groups, scores were low-average for 4 of 5 testing domains for the HIV-infected subjects and average for all 5 in the controls, and \% of HIV-infected subjects with scores classified as "low average" or below was higher than in the controls. Variables most associated with neurocognitive performance
\end{abstract}

Correspondence and reprints: Allison Ross Eckard, MD, Associate Professor of Pediatrics and Medicine, Divisions of Infectious Diseases, Medical University of South Carolina, 135 Rutledge Ave, Suite 1217, Charleston, SC 29425; Phone: 843-792-9909; Fax: 843-792-5127; eckarda@musc.edu.

ClinicalTrials.gov Identifier: NCT01523496

Conflicts of Interest: ARE has received research funding from Bristol-Myers Squibb, Cubist Pharmaceuticals, and GlaxoSmithKline and has served as an advisor and speaker for Gilead. GAM serves as a consultant for Bristol-Myers Squibb, ViiV/GlaxoSmithKline, Gilead, Pfizer, and ICON, and has received grant funding from Bristol-Myers Squibb, ViiV/GlaxoSmithKline, and Gilead. All others declare no conflicts of interest. 
among HIV-infected subjects included activated CD4+ T-cells (\% CD4+CD38+HLA-DR), monocyte activation (soluble CD14), HIV duration, age and sex.

Conclusions-HIV-infected youth on cART with virologic suppression show subtle evidence of neurocognitive impairment compared to healthy controls, and increased immune activation appears to play a role. Additional studies are needed to develop strategic interventions beyond cART to potentially improve neurocognitive performance and/or minimize further impairment in this vulnerable population.

\section{Keywords}

HIV; neurocognitive impairment; HAND; immune activation; adolescents; pediatrics

\section{INTRODUCTION}

The general term describing the spectrum of neurocognitive dysfunction seen in HIVinfected individuals is referred to as HIV-associated neurocognitive disorders (HAND). The overall reported prevalence of HAND varies from $25-60 \%$, ranging from mild to severe, and occurs in both children and adults ${ }^{1,2}$. The neurocognitive domains that are affected include processing speed, attention, executive functioning, expressive language, memory, visuospatial, and motor skills ${ }^{3-12}$. Combination antiretroviral therapy (cART) has dramatically affected the HIV pandemic by reducing the incidence of AIDS-related morbidity and mortality, including the most severe forms of HAND (e.g. AIDS-related dementia). The prevalence of milder forms of HAND, however, has actually increased in HIV-infected adults in the post-cART era ${ }^{13,14}$. Multiple studies demonstrate that even adults with well-controlled HIV infection develop HAND, suggesting that antiretrovirals (ARVs) alone are not enough to prevent and/or treat this complication ${ }^{14-16}$. Given the increased life expectancy among HIV-infected individuals, the development of HAND has serious implications for long-term quality of life ${ }^{13}$

HIV-associated neurocognitive disorders are proposed to be partly due to increased immune activation that causes neural damage ${ }^{17,18}$. HIV-infected monocytes give rise to macrophages which cross the blood-brain barrier. These macrophages are believed to serve as the predominant source of $\mathrm{HIV}$ in the CNS and are capable of producing large quantities of infectious virions in untreated HIV infection ${ }^{19}$. Even with cART, however, some studies have demonstrated that HIV persists in monocytes and has significant reactivation potential ${ }^{20}$. This potential reservoir for latent virus production may produce a low-level viral replication that causes increased immune activation even in individuals without detectable virus in blood and contributes to HAND ${ }^{21}$. For example, monocyte activation, as evidenced by expression of the CD14+ and CD16+ double-positive phenotype, as well as increased plasma levels of soluble CD14 and CD16 (sCD14, sCD16) are associated with impaired neurocognitive test performance in HIV-infected adults ${ }^{22-28}$. Similarly, these activated cells of the immune system release pro-inflammatory cytokines and other neurotoxic molecules that likely also contribute to neuronal injury ${ }^{29}$.

Like the adult population, most HIV-infected youth are living for many decades with the increased access to potent cART; however, HIV infection may still negatively impact their 
neurodevelopment ${ }^{7}$. In contrast to adults who possess a fully mature and myelinated nervous system, children and young adults are at a particularly increased risk for central nervous system (CNS) insults ${ }^{8}$. In fact, data suggest that brain development continues well into the third decade of life ${ }^{30}$, and thus, data from adult HIV populations cannot be automatically extrapolated to this younger population. To date, however, few studies have investigated neurocognitive impairment and immune activation in the pediatric HIV population and reported rather conflicting data ${ }^{31,32}$.

Thus, the primary purpose of this study was to comprehensively investigate the relationship between neurocognitive performance and markers of T-lymphocyte and monocyte immune activation among HIV-infected youth on cART with virologic suppression. The secondary objective included investigating the differences in neurocognitive performance among this HIV-infected population compared to a healthy control group. We hypothesized that higher levels of immune activation would be associated with poorer neurocognitive performance in HIV-infected youth and that this population would have worse neurocognitive performance compared to healthy controls. These mediators of HIV-related CNS injury may serve as biomarkers which would allow us to identify those at highest risk for this complication and to potentially develop strategic interventions beyond cART in this vulnerable population.

\section{METHODS}

\section{Study Design/Population}

This was a prospective, cross-sectional study, investigating levels of immune activation and relationships to neurocognitive performance in HIV-infected youth and healthy controls. For the HIV-infected group, subjects were 8-26 years of age with documented HIV-1 infection on stable cART for $\geq 12$ weeks prior to enrollment with $\geq 6$ months cumulative duration of ART, and HIV-1 RNA blood level $<1,000$ copies $/ \mathrm{mL}$. Controls were recruited from similar geographic and socioeconomic areas as the HIV-infected group, thereby decreasing the risk of potential confounders, and with an aim of achieving a group with similar characteristics as the HIV-infected subjects in terms of sex, race, and age. Documentation of absence of HIV infection was obtained in controls $\geq 13$ years of age prior to study inclusion with OraQuick Advance Rapid HIV Test (OraSure Technologies, Inc., Bethlehem, PA, USA). Exclusion criteria for both groups included any diagnosis of encephalopathy (past or present), cerebral palsy, or other known documented neurologic deficit, acute illness or inflammatory condition unless complete resolution $\geq 30$ days prior to enrollment, malignancy, or medication use (e.g. chemotherapy agents, systemic steroids) which could affect results. A previous diagnosis of a mental health disorder, such as depression, was not exclusionary for either group.

The study was reviewed and approved by the Institutional Review Boards of Emory University and Grady Health System. All parents or legal guardians and subjects $\geq 18$ years of age gave written informed consent to participate in the study. Subjects aged 17 years of age signed a written consent along with their parent or legal guardian. Subjects between the ages of 8-10 years gave verbal assent and those 11-16 years gave written assent. 


\section{Study Assessments}

Clinical and laboratory evaluations-For both groups, relevant data were obtained by questionnaire, including demographics, current and past medical and mental health history, drug habits, educational/vocational and employment background, and household socioeconomic information. Information was also collected from the HIV-infected subjects' medical records including past and current medical diagnoses, CD4+ nadir, detailed past and current ARV and non-ARV medication use, HIV diagnosis date, and acquisition method (perinatal or horizontal). Laboratory data from the HIV-infected subjects' routine HIV monitoring visits were recorded, including CD4+ cell count and HIV-1 RNA level.

Neurocognitive assessment-Neurocognitive performance was evaluated with the well-validated Wechsler Intelligence Scale ${ }^{33-35}$. The Wechsler Intelligence Scale for Children-Fourth Edition (WISC-IV) was utilized for subjects between 8-16 years old, and the Wechsler Adult Intelligence Scale-Fourth Edition (WAIS-IV) was utilized for subjects $\geq 17$ years old. These directly comparable test batteries derive a full-scale composite intelligence quotient score (FSIQ) as well as individual scores in the subcategories of verbal comprehension, perceptual reasoning, working memory, and processing speed. These scores are standardized based on the U.S. population (mean $=100$; standard deviation $(\mathrm{SD})=15$ ). In general, scores are classified as very superior ( $>130)$, superior (120-129), high average (110-119), average (90-109), low average (80-89), borderline (70-79), extremely low ( 699). Psychological assessments were performed by independently-licensed psychologists who were blinded to subjects' HIV serostatuses.

Immune activation assessment-CD4+ and CD8+ T-cell and monocyte activation were assessed via flow cytometry. All flow cytometry staining was performed on cryopreserved peripheral blood mononuclear cells that were thawed in a $37^{\circ} \mathrm{C}$ water bath and used immediately. Multi-parametric flow cytometric analysis was performed on these cells according to standard procedures, and the following panel of fluorochrome-labeled antibodies was used: anti-CD3-Alexa 700 (clone SP34-2), anti-CD8-allophycocyanin (APC)-Cy7 (clone SK1), anti-CD16-APC (clone 3G8), anti-CD38-phycoerythrin (PE) (clone HIT2), anti-CD86-fluroescein isothiocyanate (FITC) (clone FUN-1), and anti-HLADR-peridinin chlorophyll protein (PerCP)-Cy5.5 (clone G46-6) (BD Bioscience, San Jose, CA), anti-CD4-Pacific Blue (clone OKT4), and anti-CD20-Brilliant Violet 650 (clone 2H7) (Biolegend, San Diego, CA), anti-CD279 (PD-1)-PE-Cy7 (clone J105) (eBioscience, San Diego, CA), anti-CD14-ECD (RMO52) (Beckman Coulter, Brea, CA), and Live/Dead Fixable Aqua (Invitrogen, Carlsbad, CA). Flow cytometric acquisition was performed on an LSRII cytometer driven by FACS DiVa software and analyzed using FlowJo software (Treestar, Ashland, OR).

Monocytes were identified by size and granularity, then as CD3-, CD20-, CD8-, CD4dim before being categorized into subsets by CD14 and CD16 expression: CD14+CD16(classical), CD14+CD16+ (pro-inflammatory), and CD14dimCD16+ (non-classical/ patrolling). T-cells were identified by size and granularity, as well as CD3+ and CD4+ or CD8+. T-cell activation levels were assessed by CD38 and HLA-DR expression. 
Plasma levels of soluble markers of immune activation were also measured, including sCD14 and sCD16, by enzyme-linked immunosorbent assay (ELISA) using Human CD14 DuoSet (R\&D Systems, Minneapolis, MN) and anti-CD16 antibody (Abcam, Cambridge, MA), respectively. Median intra-assay and inter-assay coefficients of variance were $<9 \%$ for both assays.

\section{Statistical Analysis}

Subject characteristics are described by study group. Continuous measures are described by median/quartile $(\mathrm{Q})$ 1, 3, and nominal variables are described with frequency/percentage. Normally-distributed variables were compared using Student's $t$-tests, and non-normallydistributed variables were compared using Wilcoxon rank sum tests.

Correlations between WISC/WAIS-IV scores and variables of interest within the HIVinfected group were assessed using Spearman correlation coefficients for continuous variables. Appropriate two-sample tests were used to assess marker differences in subgroups for dichotomous variables (e.g. male vs. female). Multiple linear regression was used to explore possible associations between neurocognitive performance scores and variables which showed the most significant associations and/or potential relevance.

Evaluation of the relationship between neurocognitive performance and markers of immune activation was considered exploratory; no power calculations were determined. Statistical analysis was conducted using SAS 9.2 (The SAS Institute, Cary, NC). Statistical significance was set at the 0.05 level.

\section{RESULTS}

\section{Subject Characteristics}

A total of 66 subjects were enrolled, including $45 \mathrm{HIV}$-infected subjects and 21 healthy controls. Table 1 shows subject demographic and clinical characteristics. HIV-infected and control subjects were well-matched in terms of sociodemographic factors. All but two HIVinfected subjects had an HIV-1 RNA level $<80$ copies $/ \mathrm{mL}$, which was the highest cut-off among the different assays used in the clinical laboratories to define an "undetectable" HIV-1 RNA. These two subjects had an HIV-1 RNA level of 90 and 630 copies $/ \mathrm{mL}$, respectively.

Markers of CD4+ and CD8+ T-cell activation were higher in the HIV-infected subjects compared to controls. There were no differences in proportions of inflammatory (CD14+CD16+) or patrolling (CD14dimCD16+) monocytes between groups; however, plasma levels of both sCD14 and sCD16 were higher in the HIV-infected subjects.

\section{Neurocognitive Testing}

There were no significant differences in the median (Q1, Q3) scores between the HIVinfected subjects and healthy controls for verbal comprehension (HIV+: $89(77,96)$ vs. controls 91 (76, 106); $\mathrm{P}=0.33)$, perceptual reasoning (HIV+: 88 (79, 100) vs. controls: 92 (81, 104); $\mathrm{P}=0.44)$, processing speed (HIV+: 97 (84, 102) vs. controls: $94(89,108)$; $\mathrm{P}=0.44$ ), and FSIQ (HIV+: 87 (77, 95) vs. controls: 94 (80, 110); $\mathrm{P}=0.19)$ (Figure 1). There 
was a trend toward significance for working memory (HIV+: $89(77,99)$ vs. controls: 97 $(86,113) ; \mathrm{P}=0.05)$. In the HIV-infected group, neurocognitive scores were in the lowaverage range (80-89) for 4 of 5 domains. In controls, all 5 scores were in the average range (90-109). Similarly, \% of HIV-infected subjects with scores $\$ 89$ and thus classified in the "low average" (80-89), "borderline" (70-79) or "extremely low" ( 69 ) ranges was higher than in the healthy controls for four of the domains [verbal comprehension (HIV+: $53 \%$ vs. controls: $43 \%$; $\mathrm{P}=0.43$ ), perceptual reasoning (HIV+: $51 \%$ vs. controls: $38 \%$; $\mathrm{P}=0.32$ ), working memory: HIV+: $56 \%$ vs. controls: $28 \%$; $\mathrm{P}=0.04$, FSIQ (HIV+: $62 \%$ vs. controls: $43 \% ; \mathrm{P}=0.14)$. Only working memory reached statistical significance. Percentage of subjects with a score $\$ 89$ was higher among the controls for processing speed but was not statistically significant (HIV+: $36 \%$ vs. controls: $38 \%$; $\mathrm{P}=0.84$ ).

\section{Relationships between Neurocognitive Scores and Variables of Interest}

Associations between neurocognitive performance and variables of interest among HIVinfected subjects are shown in Table 2. A significant negative correlation was found between HIV duration and 3 of the 5 categories (verbal comprehension, working memory and FSIQ) (Figure 2); whereas, ART duration had a significant negative correlation with only working memory and FSIQ. None of the neurocognitive performance categories were significantly correlated with age, CD4 nadir, CD8+ activation (\% CD8+CD38+HLA-DR+), inflammatory (CD14+CD16+) or patrolling (CD14dimCD16+) monocytes, or sCD16. On the other hand, $\%$ of T-cells expressing the activated phenotype, CD38+HLA-DR+, was negatively correlated with verbal comprehension. Likewise, a higher plasma level of sCD14, a soluble marker of monocyte activation, was negatively associated with both verbal comprehension and FSIQ (Figure 2).

Subjects with perinatal transmission of HIV had significantly lower neurocognitive scores for the same domains as were associated with HIV duration: verbal comprehension, working memory and FSIQ. Males had significantly higher scores compared to females for verbal comprehension, perceptual reasoning, and FSIQ, and non-blacks had a significantly higher score for processing speed. Subjects who reported current marijuana use also had significantly higher scores for working memory, processing speed, and FSIQ. On the other hand, scores for subjects with a prior AIDS diagnosis were not statistically different than scores from subjects without a diagnosis.

Multivariable regression models were created for each of the 5 testing categories, using the same variables for each model (\% CD4+CD38+HLA-DR, sCD14, HIV duration, age, male sex, and current marijuana use (Table 3)). For verbal comprehension, sCD14 was the only significant variable in the model with HIV duration trending toward significant, where a lower SCD14 concentration and a shorter HIV duration was associated with a higher score. For processing speed, $\%$ CD4+CD38+HLA-DR was the only variable significant in the final model, where a lower $\%$ of CD4 activated cells was associated with a higher score, and current marijuana use trended toward significant, where current use was associated with a higher score. Male sex and a younger age were significantly associated with a higher score for perceptual reasoning, and HIV duration was the only variable significant for both working memory and FSIQ (i.e. a shorter HIV duration was associated with higher scores). 
For all models, mode of HIV transmission (perinatal vs. horizontal) was substituted in place of HIV duration and the analysis re-done. In all cases, HIV duration was statistically stronger, and mode of transmission was not significantly significant in any model (data not shown).

The beta-coefficients +/- standard errors of selected variables from the regression models were also plotted relative to a null effect to allow comparison across the neurocognitive testing categories (Figure 3).

\section{DISCUSSION}

In this current study, we investigated the relationship between neurocognitive performance and markers of T-lymphocyte and monocyte immune activation among HIV-infected youth on cART with virologic suppression. HIV-infected youth demonstrated increased immune activation compared to controls with higher peripheral concentrations of CD4+ and CD8+ Tlymphocyte activation. Soluble CD14 and sCD16 were also higher in the HIV-infected group, although monocyte subpopulations did not differ between groups. In univariate analysis, sCD14 and \% CD4+CD38+HLA-DR+ were both inversely correlated with verbal comprehension in the HIV-infected group, and sCD14 was also inversely correlated with FSIQ. In the multivariable regression models, a lower sCD14 was independently associated with a higher verbal comprehension score, and a lower \% CD4+CD38+HLA-DR+ was independently associated with a higher processing speed score.

Applying a well-validated comprehensive neurocognitive testing battery, we did not detect any statistically significant differences in scores between the HIV-infected group and the healthy uninfected controls. However, there were indications that the HIV-infected subjects had subtle impairments compared to the controls. For example, scores for the HIV-infected subjects were all in the low-average range compared to the average range among the controls, and the proportion of HIV-infected subjects in the low-average or below range was higher than the controls in all categories, but processing speed. The lack of statistically significant findings in all domains except working memory may reflect a small sample size; however, it may also demonstrate some improvement or a change in the pattern of neurocognitive impairment that accompanies cART-induced virologic suppression. This finding is supported by data from other studies in the adult and pediatric HIV populations ${ }^{16,36}$.

While our findings suggest that cART may modestly improve or change the pattern of neurocognitive impairment, it does not appear to restore neurocognitive function to normal. Thus, other etiologies are likely at play in the setting of virologic suppression. In HIVinfected adults, the increased immune activation associated with HIV infection has been implicated as a primary factor ${ }^{19-21}$. It is postulated that infected monocytes cross the bloodbrain barrier and establish reservoirs which serve as sources of ongoing CNS viral replication and immune activation ultimately leading to neural damage ${ }^{17,19,37}$. Monocyte activation, as evidenced by increased expression of the CD14+ and CD16+ phenotype and increased plasma levels of sCD14 and sCD16, has been associated with impaired neurocognitive performance in HIV-infected adults. 
Little data exist in HIV-infected youth. To date, only one study has investigated T-cell activation and neurocognitive performance in HIV-infected children ${ }^{32}$, and only one study has investigated activated monocyte phenotypes. ${ }^{38}$ Both studies suggested that immune activation may actually provide a neuroprotective effect in the pediatric population. This is in stark contrast to the data in the adult population that shows deleterious effects of immune activation on the $\mathrm{CNS}^{39-41}$. Both pediatric studies evaluated relatively young children (median age 6-7 years) with perinatal HIV who had only been on cART for a relatively short period of time or who were not on therapy. Given that our HIV-infected population was considerably older (median age 19 years) and included horizontally-infected youth, it is likely that our finding of increased immune activation and neurocognitive impairment more closely resembles that seen in the adult population. Future studies specifically investigating perinatally-infected young adults would perhaps help elucidate the pathogenesis behind this discrepancy.

Interestingly, we did not find any significant correlations with the monocyte cell phenotypes associated with immune activation and any of the neurocognitive testing domains. The reason for the lack of between-group differences in monocyte subpopulations is unclear, but may be related to the fact that HIV-infected subjects were virologically-suppressed, on stable cART, and had subtle forms of HAND. This finding is supported by the fact that the highest concentrations of activated CD14+CD16+ monocytes have been found in patients with HIVassociated dementia rather than milder forms of neurocognitive impairment and examined subjects who were not on adequate ART or virologically-suppressed. ${ }^{26,42}$ Likewise, increased sCD14 levels have been associated with neurocognitive impairments in HIVinfected adults ${ }^{25,28}$.

Multivariable regression analyses revealed that HIV duration was arguably the variable most associated with neurocognitive performance, where a longer HIV duration was associated with impaired neurocognitive impairment, consistent with studies in adults ${ }^{43}$. One could postulate here, however, that HIV duration is merely a surrogate marker for perinatal HIV transmission since this group in general would have a longer duration of disease. However, mode of transmission was not significant in any regression model. One reason for this discrepancy may be because, in general, continuous variables have more statistical power than dichotomous variables. On the other hand, HIV duration may merely be more associated with neurocognitive functioning than mode of transmission.

Similarly, if cART-induced virologic suppression is associated with improved neurocognitive performance, then one would expect that a longer duration of cART would be associated with higher scores (which is the opposite of what we observed). This is probably because the cART variable suffered confounding from other factors, such as HIV duration. Likewise, males showed better scores than females in several domains in bivariate analysis, but this difference was not significant in the multivariable regression models. Males were statistically older than females with a shorter HIV and ARV duration (data not shown). This reflects the high proportion of males in our study who acquired HIV via high-risk homosexual activity later in life and have had HIV for a relatively short period of time. 
Another interesting finding was the significant, positive association in HIV-infected subjects between marijuana use and 3 neurocognitive domains (and a trend toward significance in one of the regression models). Historically, marijuana use has been associated with impaired memory and other measures of neurocognitive functioning 44,45 . However, recent evidence has emerged which indicates that agonists of the cannabinoid receptor type $2\left(\mathrm{CB}_{2}\right)$ have therapeutic potential in a variety of pathologic conditions involving chronic inflammation and immune activation, including HIV-1 infection ${ }^{46-50}$. It is unclear from our data if marijuana use actually provided a neuroprotective effect or if it too showed confounding from other factors. Further studies are warranted to investigate this potential therapeutic role in the HIV-infected population ${ }^{51}$.

Despite the interesting findings, our study suffered from some limitations worth mentioning. These include a relatively small number of HIV-infected subjects which may have misrepresented the actual prevalence of neurocognitive impairment in this population, and due to the cross-sectional design, causality cannot be implied from the associations demonstrated. The fact that some variables were associated with some but not all neurocognitive domains may also reflect these same issues. Likewise, despite well-matched groups, it is possible that results were confounded by unmeasured environmental factors. Our study population was also mostly African American, and, thus, the generalizability to other races cannot be assumed. Finally, only peripheral blood was utilized. Many studies on the pathophysiology of HAND in adults are now focusing on measurement of biomarkers in the CSF as a potentially more accurate representation of immune activation in the CNS.

A growing body of evidence supports the high prevalence, etiology, and implications of HAND in the adult HIV-infected population, but few studies have evaluated this potentiallydevastating complication in HIV-infected youth in the post-cART era. The current goal of HIV treatment is long-term virologic suppression to decelerate disease progression and minimize the risk of co-morbidities. Thus, we chose to focus our evaluation on HIV-infected youth on cART with undetectable or very low-level viremia. This study provides evidence of below-average performance among virologically-suppressed HIV-infected youth on measures of neurocognitive function with heightened immune activation likely playing a role. To date, our study is the first to demonstrate the relationship between increased immune activation and worse neurocognitive performance in HIV-infected youth. As youth ages 13-24 years old comprise one of the fastest growing populations of newly-infected HIV-infected individuals in the United States ${ }^{52}$, ramifications of unrecognized HAND could be immense. Thus, more studies investigating the etiology, pathogenesis, and possible treatments of neurocognitive impairment in this younger population are urgently needed. Targeting this vulnerable population offers a unique opportunity to identify those most at risk for poor neurocognitive outcomes and develop potential strategies to mitigate neurological damage while neurodevelopment is still occurring.

\section{Acknowledgments}

Sources of Support: This work was made possible by the National Institute of Child Health and Development at the National Institutes of Health [K23 HD069199 to ARE; R01 HD070490 to GAM; K12 HD072245 to AC], Case Western Reserve University's Center for AIDS Research (P30 AI36219), Emory University's Center for AIDS Research (P30 AI050409), Emory+Children's Pediatric Research Center (Biomarkers, Immunology and Flow 
Cytometry Cores), Clinical and Translational Science Award and the Clinical and Translational Science Collaborative of Cleveland from the National Center for Advancing Translational Sciences (NCATS) component of the National Institutes of Health and NIH roadmap for Medical Research (UL1TR000439). The content is solely the responsibility of the authors and does not necessarily represent the official views of the National Institutes of Health.

\section{References}

1. Van Rie AHP, Dow A, Robertson K. Neurologic and neurodevelopmental manifestations of pediatric HIV/AIDS: A global perspective. European journal of paediatric neurology : EJPN : official journal of the European Paediatric Neurology Society. 2007; 11:1-9. [PubMed: 17137813]

2. Letendre S. Central nervous system complications in HIV disease: HIV-associated neurocognitive disorder. Top Antivir Med. Nov; 2011 19(4):137-142. [PubMed: 22156215]

3. Schouten J, Cinque P, Gisslen M, Reiss P, Portegies P. HIV-1 infection and cognitive impairment in the cART era: a review. Aids. Mar 13; 2011 25(5):561-575. [PubMed: 21160410]

4. Drotar D, Olness K, Wiznitzer M, et al. Neurodevelopmental outcomes of Ugandan infants with HIV infection: an application of growth curve analysis. Health Psychol. Mar; 1999 18(2):114-121. [PubMed: 10194046]

5. Gay CL, Armstrong FD, Cohen D, et al. The effects of HIV on cognitive and motor development in children born to HIV-seropositive women with no reported drug use: birth to 24 months. Pediatrics. Dec; 1995 96(6):1078-1082. [PubMed: 7491224]

6. Knight WG, Mellins CA, Levenson RL Jr, Arpadi SM, Kairam R. Brief report: effects of pediatric HIV infection on mental and psychomotor development. J Pediatr Psychol. Dec; 2000 25(8):583587. [PubMed: 11085762]

7. Koekkoek S, de Sonneville LM, Wolfs TF, Licht R, Geelen SP. Neurocognitive function profile in HIV-infected school-age children. European journal of paediatric neurology : EJPN : official journal of the European Paediatric Neurology Society. Jul; 2008 12(4):290-297. [PubMed: 17950012]

8. Allen, ABJM., Forsyth, B. Pediatric HIV/AIDS. In: Davis, A., editor. The Handbook of Pediatric Neuropsychology. New York: Springer Publishing Company, LLC; 2011. p. 865-876.

9. Bisiacchi PS, Suppiej A, Laverda A. Neuropsychological evaluation of neurologically asymptomatic HIV-infected children. Brain Cogn. Jun-Aug;2000 43(1-3):49-52. [PubMed: 10857661]

10. Tardieu M, Mayaux MJ, Seibel N, et al. Cognitive assessment of school-age children infected with maternally transmitted human immunodeficiency virus type 1. J Pediatr. Mar; 1995 126(3):375379. [PubMed: 7869195]

11. Havens J, Whitaker A, Feldman J, Alvarado L, Ehrhardt A. A controlled study of cognitive and language function in school-aged HIV-infected children. Ann N Y Acad Sci. Oct 29.1993 693:249-251. [PubMed: 8267269]

12. Llorente, AMLC., Satz, P. Neurobehavioral and Neurodevelopmental Sequelae Associated with Pediatric HIV Infection. In: Reynolds, CRF-JE., editor. Handbook of Clinical Child Neuropsychology. 3. New York: Springer Publishing Company, LLC; 2009. p. 635-669.

13. Rumbaugh JA, Steiner J, Sacktor N, Nath A. Developing neuroprotective strategies for treatment of HIV-associated neurocognitive dysfunction. Futur HIV Ther. 2008; 2(3):271-280. [PubMed: 19774095]

14. Simioni S, Cavassini M, Annoni JM, et al. Cognitive dysfunction in HIV patients despite longstanding suppression of viremia. Aids. Jun 1; 2010 24(9):1243-1250. [PubMed: 19996937]

15. Robertson KR, Smurzynski M, Parsons TD, et al. The prevalence and incidence of neurocognitive impairment in the HAART era. Aids. Sep 12; 2007 21(14):1915-1921. [PubMed: 17721099]

16. Heaton RK, Franklin DR, Ellis RJ, et al. HIV-associated neurocognitive disorders before and during the era of combination antiretroviral therapy: differences in rates, nature, and predictors. Journal of neurovirology. Feb; 2011 17(1):3-16. [PubMed: 21174240]

17. Ellis RJ, Calero P, Stockin MD. HIV infection and the central nervous system: a primer. Neuropsychol Rev. Jun; 2009 19(2):144-151. [PubMed: 19415500]

18. Valcour V, Sithinamsuwan P, Letendre S, Ances B. Pathogenesis of HIV in the central nervous system. Curr HIV/AIDS Rep. Mar; 2011 8(1):54-61. [PubMed: 21191673] 
19. Gras G, Kaul M. Molecular mechanisms of neuroinvasion by monocytes-macrophages in HIV-1 infection. Retrovirology. 2010; 7:30. [PubMed: 20374632]

20. Lambotte O, Taoufik Y, de Goer MG, Wallon C, Goujard C, Delfraissy JF. Detection of infectious HIV in circulating monocytes from patients on prolonged highly active antiretroviral therapy. J Acquir Immune Defic Syndr. Feb 1; 2000 23(2):114-119. [PubMed: 10737425]

21. Kapetanovic S, Leister E, Nichols S, et al. Relationships between markers of vascular dysfunction and neurodevelopmental outcomes in perinatally HIV-infected youth. Aids. Jun 19; 2010 24(10): 1481-1491. [PubMed: 20539091]

22. Kaul M, Lipton SA. Mechanisms of neuroimmunity and neurodegeneration associated with HIV-1 infection and AIDS. J Neuroimmune Pharmacol. Jun; 2006 1(2):138-151. [PubMed: 18040780]

23. Fischer-Smith T, Croul S, Sverstiuk AE, et al. CNS invasion by CD14+/CD16+ peripheral bloodderived monocytes in HIV dementia: perivascular accumulation and reservoir of HIV infection. $\mathrm{J}$ Neurovirol. Dec; 2001 7(6):528-541. [PubMed: 11704885]

24. Khayat D, Soubrane C, Andrieu JM, et al. Changes of soluble CD16 levels in serum of HIVinfected patients: correlation with clinical and biologic prognostic factors. J Infect Dis. Mar; 1990 161(3):430-435. [PubMed: 2138203]

25. Lyons JL, Uno H, Ancuta P, et al. Plasma sCD14 is a biomarker associated with impaired neurocognitive test performance in attention and learning domains in HIV infection. $\mathrm{J}$ Acquir Immune Defic Syndr. Aug 15; 2011 57(5):371-379. [PubMed: 21646912]

26. Pulliam L, Gascon R, Stubblebine M, McGuire D, McGrath MS. Unique monocyte subset in patients with AIDS dementia. Lancet. Mar 8; 1997 349(9053):692-695. [PubMed: 9078201]

27. Kamat A, Lyons JL, Misra V, et al. Monocyte activation markers in cerebrospinal fluid associated with impaired neurocognitive testing in advanced HIV infection. J Acquir Immune Defic Syndr. Jul 1; 2012 60(3):234-243. [PubMed: 22569268]

28. Kamat A, Misra V, Cassol E, et al. A plasma biomarker signature of immune activation in HIV patients on antiretroviral therapy. PLoS One. 2012; 7(2):e30881. [PubMed: 22363505]

29. Marcotte TD, Deutsch R, Michael BD, et al. A concise panel of biomarkers identifies neurocognitive functioning changes in HIV-infected individuals. Journal of neuroimmune pharmacology : the official journal of the Society on NeuroImmune Pharmacology. Dec; 2013 8(5):1123-1135. [PubMed: 24101401]

30. Lebel C, Beaulieu C. Longitudinal development of human brain wiring continues from childhood into adulthood. The Journal of neuroscience : the official journal of the Society for Neuroscience. Jul 27; 2011 31(30):10937-10947. [PubMed: 21795544]

31. Kapetanovic S, Griner R, Zeldow B, et al. Biomarkers and neurodevelopment in perinatally HIVinfected or exposed youth: a structural equation model analysis. Aids. Jan 28; 2014 28(3):355364. [PubMed: 24670521]

32. Kapetanovic S, Aaron L, Montepiedra G, Burchett SK, Kovacs A. T-cell activation and neurodevelopmental outcomes in perinatally HIV-infected children. Aids. May 15; 2012 26(8): 959-969. [PubMed: 22382148]

33. Wechsler, D. WAIS-IV Administration and Scoring Manual. San Antonio: The Psychological Corporation; 2008a.

34. Wechsler, D. WAIS-IV Technical and Interpretive Manual. San Antonio: The Psychological Corporation; 2008b.

35. Wechsler, D. Wechsler Intelligence Scale for Children-Fourth Edition. San Antonio: Harcourt Assessment, Inc; 2003.

36. Crowell CS, Huo Y, Tassiopoulos K, et al. Early viral suppression improves neurocognitive outcomes in HIV-infected children. Aids. Jan 28; 2015 29(3):295-304. [PubMed: 25686678]

37. Shiramizu B, Ananworanich J, Chalermchai T, et al. Failure to clear intra-monocyte HIV infection linked to persistent neuropsychological testing impairment after first-line combined antiretroviral therapy. J Neurovirol. Feb; 2012 18(1):69-73. [PubMed: 22207583]

38. Ananworanich J, Kerr SJ, Jaimulwong T, et al. Soluble CD163 and monocyte populations in response to antiretroviral therapy and in relationship with neuropsychological testing among HIVinfected children. Journal of virus eradication. 2015; 1(3):196-202. [PubMed: 26835517] 
39. Grauer OM, Reichelt D, Gruneberg U, et al. Neurocognitive decline in HIV patients is associated with ongoing T-cell activation in the cerebrospinal fluid. Annals of clinical and translational neurology. Sep; 2015 2(9):906-919. [PubMed: 26401512]

40. Vassallo M, Durant J, Lebrun-Frenay C, et al. Virologically suppressed patients with asymptomatic and symptomatic HIV-associated neurocognitive disorders do not display the same pattern of immune activation. HIV medicine. Aug; 2015 16(7):431-440. [PubMed: 25981452]

41. Brew BJ, Letendre SL. Biomarkers of HIV related central nervous system disease. International review of psychiatry. Feb; 2008 20(1):73-88. [PubMed: 18240064]

42. Ziegler-Heitbrock L. The CD14+ CD16+ blood monocytes: their role in infection and inflammation. Journal of leukocyte biology. Mar; 2007 81(3):584-592. [PubMed: 17135573]

43. Bhaskaran K, Mussini C, Antinori A, et al. Changes in the incidence and predictors of human immunodeficiency virus-associated dementia in the era of highly active antiretroviral therapy. Ann Neurol. Feb; 2008 63(2):213-221. [PubMed: 17894380]

44. Crane NA, Schuster RM, Gonzalez R. Preliminary evidence for a sex-specific relationship between amount of cannabis use and neurocognitive performance in young adult cannabis users. J Int Neuropsychol Soc. Oct; 2013 19(9):1009-1015. [PubMed: 23962414]

45. Dougherty D, Mathias CW, Dawes MA, Furr RM, Charles NE, Liguori A, Shannon EE, Acheson A. Impulsivity, attention, memory, and decision-making among adolescent marijuana users. Psychopharmacology. 2013; 226(2):307-319. [PubMed: 23138434]

46. Molina PE, Amedee A, LeCapitaine NJ, et al. Cannabinoid neuroimmune modulation of SIV disease. J Neuroimmune Pharmacol. Dec; 2011 6(4):516-527. [PubMed: 21830069]

47. Molina PE, Winsauer P, Zhang P, et al. Cannabinoid administration attenuates the progression of simian immunodeficiency virus. AIDS Res Hum Retroviruses. Jun; 2011 27(6):585-592. [PubMed: 20874519]

48. Peterson PK, Gekker G, Hu S, Cabral G, Lokensgard JR. Cannabinoids and morphine differentially affect HIV-1 expression in CD4(+) lymphocyte and microglial cell cultures. J Neuroimmunol. Feb; 2004 147(1-2):123-126. [PubMed: 14741442]

49. Rom S, Persidsky Y. Cannabinoid receptor 2: potential role in immunomodulation and neuroinflammation. J Neuroimmune Pharmacol. Jun; 2013 8(3):608-620. [PubMed: 23471521]

50. Kim H, Shin AH, Thayer SA. Activation of cannabinoid type 2 receptors inhibits HIV-1 envelope glycoprotein gp120-induced synapse loss. Molecular Pharmacology. 2011; 80:357-366. [PubMed: 21670103]

51. Lutge, E., Gray, A., Siegfried, N. The Cochrane database of systematic reviews. 2013. Apr 30, 2013 The medical use of cannabis for reducing morbidity and mortality in patients with HIV/ AIDS.

52. Centers for Disease Control and Prevention. HIV Surveillance Report 2014. 26 [Accessed May 26, 2016] Published November 2015. 

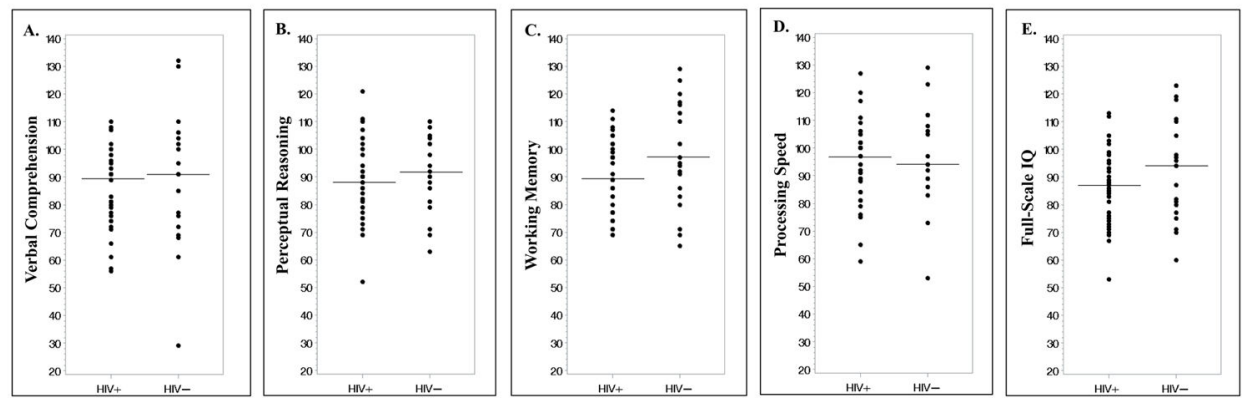

Figure 1. Neurocognitive performance by study group

These scatter plots show the distribution of neurocognitive testing scores for HIV-infected subjects compared to healthy controls for (A) verbal comprehension, (B) perceptual reasoning, $(\mathrm{C})$ working memory, (D) processing speed and (E) full-scale intelligence quotient (FSIQ). In the HIV-infected group, median scores were in the low-average range for 4 of 5 composite scores [median (Q1, Q3) verbal comprehension: 89 (77, 96); perceptual reasoning: $88(79,100)$; working memory: $89(77,99)$; FSIQ: $87(77,95)]$, and in the average range for processing speed $[97(84,102)]$. For the control subjects, median scores were in the average range for all 5 composite scores [median $(\mathrm{Q} 1, \mathrm{Q} 3)$ verbal comprehension: 91 (76, 106); perceptual reasoning: 92 (81, 104); working memory: 97 (86, 113); processing speed: 94 (89, 108); FSIQ: $94(80,110)]$. However, there were no statistically significant differences in the scores for any of the categories between the HIVinfected subjects and healthy controls. Horizontal lines denote medians. 

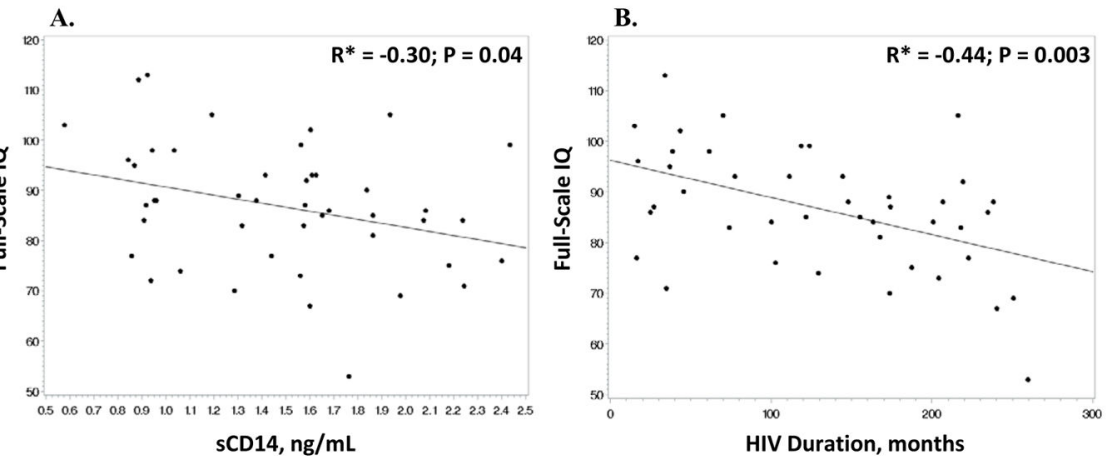

Figure 2. Correlations between full-scale IQ and significant variables Scatter plots depict the bivariate relationship between full-scale IQ and A. SCD14 and B. HIV duration for the HIV-infected subjects. Full-scale IQ was significantly negatively correlated with both sCD14 and HIV duration. *Spearman correlation coefficient. IQ, intelligence quotient; sCD14, soluble CD14. 

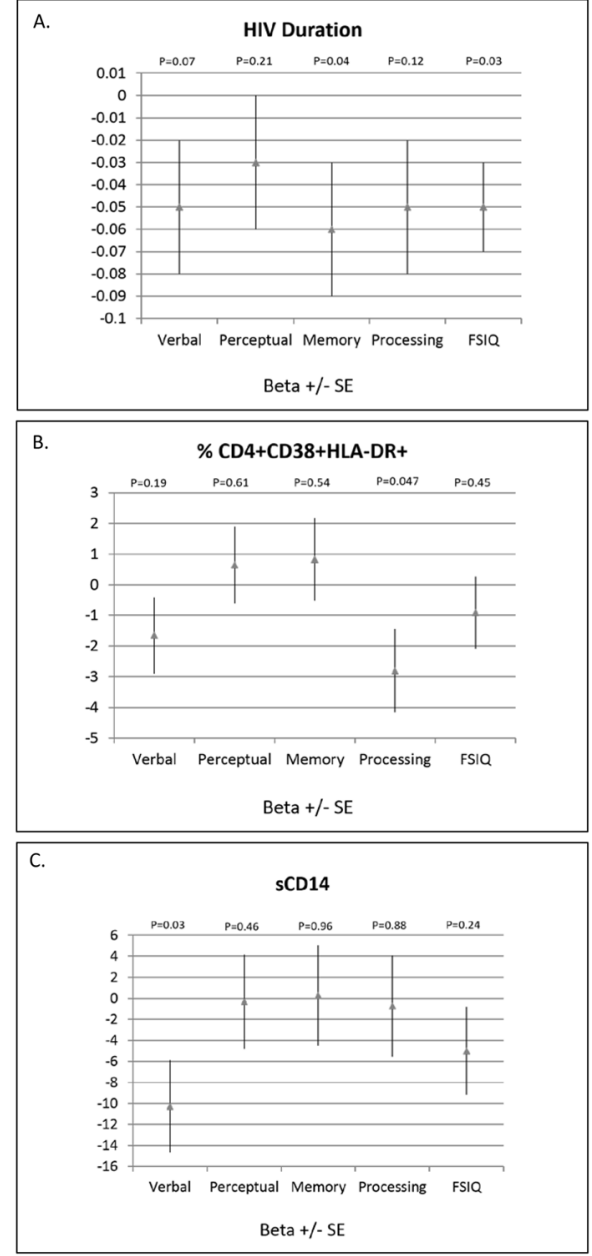

Figure 3. Comparison of the effect of selected variables on the neurocognitive scales These plots show the beta-coefficients $+/-$ standard errors relative to a null effect for selected variables from the multivariable models for ease of comparison across the neurocognitive testing categories (A. HIV duration, B. \% CD4+CD38+HLA-DR+ and C. sCD14). sCD14, soluble CD14; FSIQ, full-scale intelligence quotient. 


\section{Table 1}

Characteristics of Study Population

\begin{tabular}{|c|c|c|c|}
\hline Median (Q1, Q3) or no. (\%) & $\mathrm{HIV}+(\mathrm{N}=45)$ & Controls $(\mathrm{N}=21)$ & $\mathbf{P}$ \\
\hline \multicolumn{4}{|l|}{ A. Demographics } \\
\hline Age, years & $19.1(14.4,22.7)$ & $16.5(11.9,18.9)$ & 0.06 \\
\hline Male sex & $16(58 \%)$ & $10(48 \%)$ & 0.60 \\
\hline Black race & $40(89 \%)$ & $21(100 \%)$ & 0.17 \\
\hline Highest level of education & & & 0.69 \\
\hline Elementary-high school & $23(52 \%)$ & $13(62 \%)$ & \\
\hline High school diploma/GED & $13(30 \%)$ & $5(24 \%)$ & \\
\hline Some college & $6(14 \%)$ & $1(5 \%)$ & \\
\hline Bachelor's degree & $2(4 \%)$ & $2(10 \%)$ & \\
\hline Special education history & $9(20 \%)$ & $2(10 \%)$ & 0.48 \\
\hline Mental health disorder history & $7(16 \%)$ & $5(24 \%)$ & 0.50 \\
\hline Psychotropic medication history & $7(16 \%)$ & $0(0 \%)$ & 0.06 \\
\hline Current mental health professional care & $6(14 \%)$ & $2(10 \%)$ & 1.00 \\
\hline Current marijuana use ${ }^{*}$ & $8(18 \%)$ & $3(14 \%)$ & 1.00 \\
\hline \multicolumn{4}{|l|}{ B. HIV Variables } \\
\hline Current CD4 count, cells $/ \mathrm{mm}^{3}$ & $630(411,833)$ & -- & -- \\
\hline Nadir CD4 count, cells $/ \mathrm{mm}^{3}$ & $254(114,356)$ & -- & -- \\
\hline HIV-1 RNA level $<80$ copies/mL ${ }^{* *}$ & $43(96 \%)$ & -- & -- \\
\hline Perinatally-acquired HIV & $27(60 \%)$ & -- & -- \\
\hline Prior AIDS diagnosis & $14(41 \%)$ & -- & -- \\
\hline HIV duration, years & $10.8(3.8,17.0)$ & -- & -- \\
\hline ART duration, years & $6.5(1.8,10.9)$ & -- & -- \\
\hline PI duration, years & $6.2(1.6,11.2)$ & -- & -- \\
\hline NNRTI duration, years & $1.2(0.0,1.6)$ & -- & -- \\
\hline Current PI use & $30(67 \%)$ & -- & -- \\
\hline Current NNRTI use & $11(24 \%)$ & -- & -- \\
\hline Current integrase inhibitor use & $9(20 \%)$ & -- & -- \\
\hline \multicolumn{4}{|l|}{ C. Immune activation markers } \\
\hline$\% \mathrm{CD} 4+\mathrm{CD} 38+\mathrm{HLA}-\mathrm{DR}+{ }^{a}$ & $1.4(1.1,2.1)$ & $1.1(0.9,1.6)$ & 0.03 \\
\hline$\% \mathrm{CD} 8+\mathrm{CD} 38+\mathrm{HLA}-\mathrm{DR}+{ }^{b}$ & $3.4(2.3,5.1)$ & $1.2(0.8,2.7)$ & 0.0005 \\
\hline$\% \mathrm{CD} 14+\mathrm{CD} 16+{ }^{c}$ & $11.8(7.8,16.4)$ & $13.9(8.7,19.6)$ & 0.43 \\
\hline$\% \mathrm{CD} 14 \operatorname{dimCD} 16+{ }^{d}$ & $13.2(7.4,23.3)$ & $9.2(6.2,15.8)$ & 0.09 \\
\hline $\mathrm{sCD} 14, \mathrm{ng} / \mathrm{mL}^{e}$ & $1.6(1.0,1.8)$ & $1.2(0.9,1.3)$ & 0.01 \\
\hline $\mathrm{sCD} 16, \mathrm{ng} / \mathrm{mL}^{e}$ & $0.9(0.6,1.5)$ & $0.6(0.3,0.9)$ & 0.0498 \\
\hline
\end{tabular}


N.B. P-values with bolded font designate those $<0.05$. Data missing for 2, 3, 2, 5, 1, $11 \mathrm{HIV}+$ subjects for HIV duration, ART duration, current CD4 count, nadir CD4 count, highest level of education, prior AIDS diagnosis, respectively; Data missing for 1 control subject for special education; Data missing for $3 \mathrm{HIV}+$ subjects and 1 control subject for cellular immune activation markers;

* Defined as any use in the previous 3 months.

**

Because there were several different assays used in the clinical laboratories to measure HIV-1 RNA levels, there were varying lower limits of detection $(<20,<40,<48,<79$ and $<80$ copies/mL). $<80$ copies/mL was the highest cut-off that defined an undetectable HIV-1 RNA.

${ }^{a} \%$ of CD4+ T-cells expressing CD38 and HLA-DR;

$b_{\%}$ of CD8+ T-cells expressing CD38 and HLA-DR;

$c_{\%}$ of monocytes expressing CD14+CD16+;

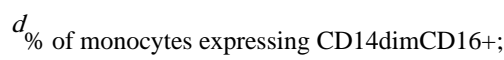

e Plasma concentration of sCD14 and sCD16, respectively.

Q, quartile; HIV, human immunodeficiency virus; GED, general educational development; ART, antiretroviral therapy; PI, protease inhibitor; NNRTI, non-nucleoside analogue reverse transcriptase inhibitor; sCD14, soluble CD14; sCD16, soluble CD16 

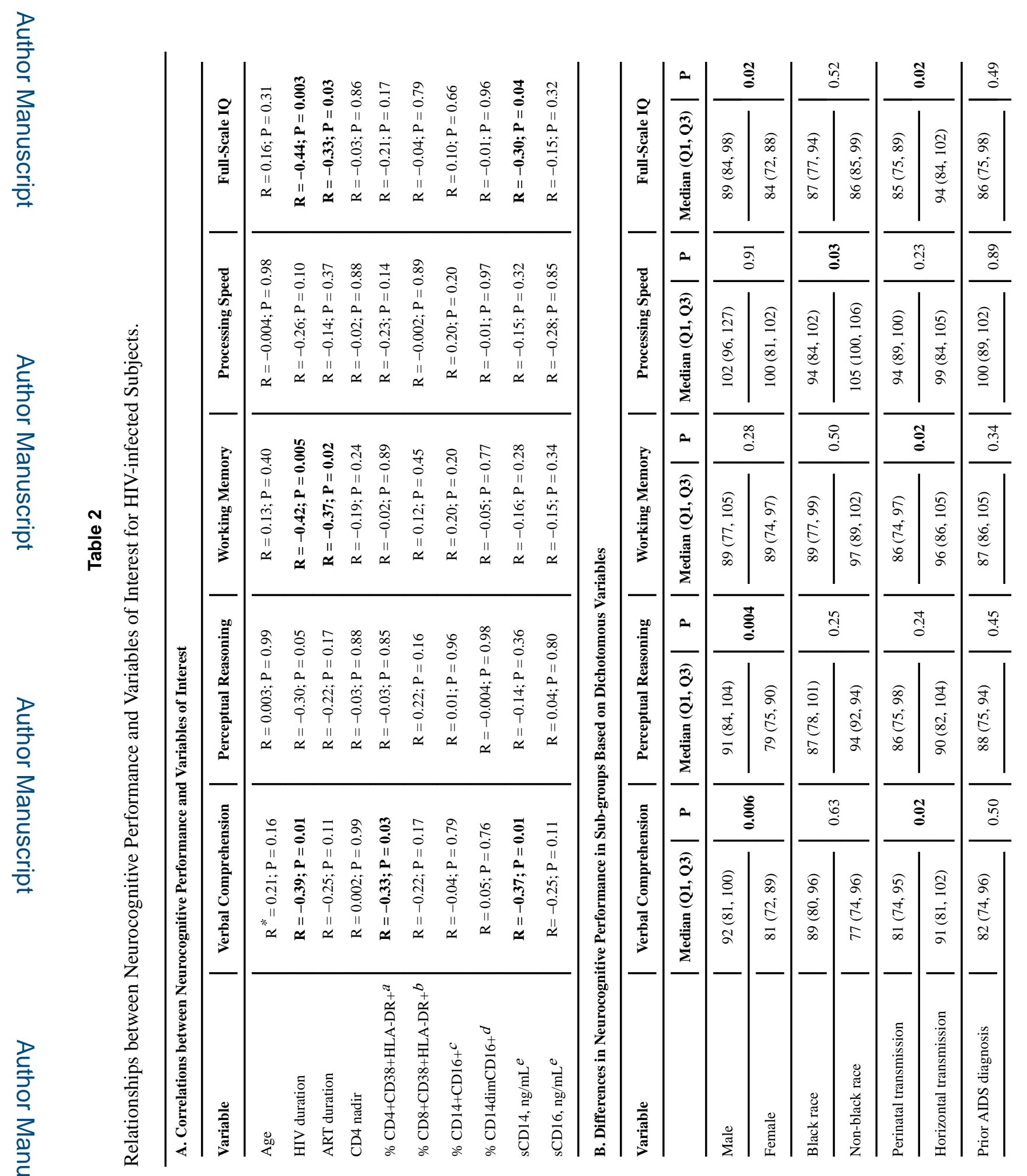

Antivir Ther. Author manuscript; available in PMC 2018 May 08. 


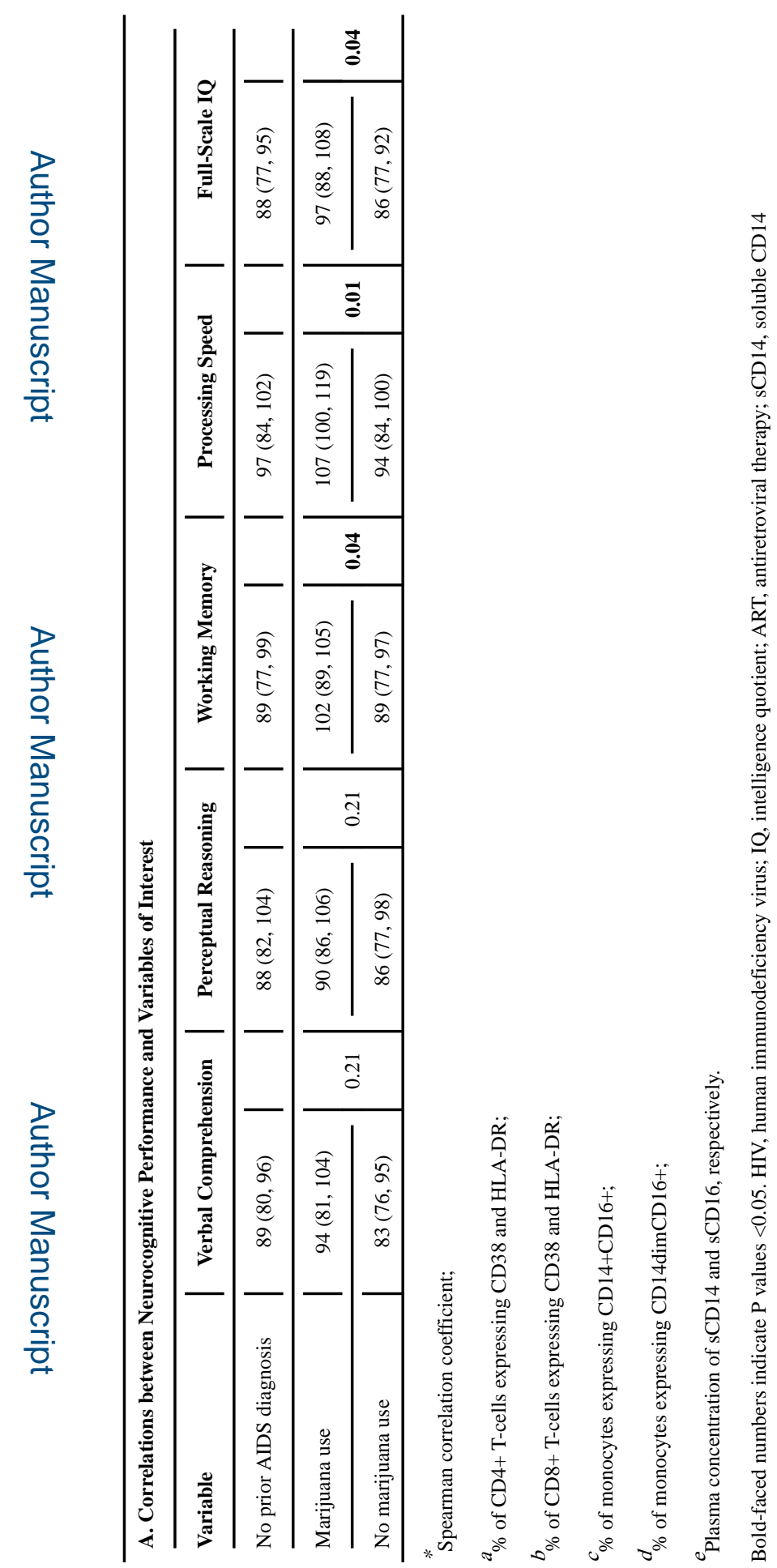

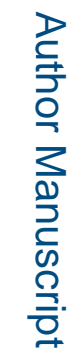




\section{Table 3}

Multivariable Regression Models for Variables Associated with Neurocognitive Performance in HIV-infected Subjects

\begin{tabular}{|c|c|c|c|}
\hline \multicolumn{4}{|l|}{ A. Verbal Comprehension } \\
\hline Variable & Beta & SE (Beta) & $\mathbf{P}$ \\
\hline$\% \mathrm{CD} 4+\mathrm{CD} 38+\mathrm{HLA}-\mathrm{DR}+{ }^{a}$ & -1.65 & 1.24 & 0.19 \\
\hline $\mathrm{sCD} 14$ & -10.3 & 4.39 & 0.03 \\
\hline HIV duration & -0.05 & 0.03 & 0.07 \\
\hline Age & -0.41 & 0.43 & 0.35 \\
\hline Sex (male vs. female) & 6.06 & 4.34 & 0.17 \\
\hline \multirow[t]{2}{*}{ Marijuana use (current use vs. no current use) } & -5.87 & 5.46 & 0.29 \\
\hline & \multicolumn{3}{|c|}{$\mathrm{R}^{2}=0.36$} \\
\hline \multicolumn{4}{|l|}{ B. Perceptual Reasoning } \\
\hline Variable & Beta & SE (Beta) & $\mathbf{P}$ \\
\hline$\% \mathrm{CD} 4+\mathrm{CD} 38+\mathrm{HLA}-\mathrm{DR}+{ }^{a}$ & 0.65 & 1.26 & 0.61 \\
\hline sCD14 & -0.34 & 4.47 & 0.46 \\
\hline HIV duration & -0.03 & 0.03 & 0.21 \\
\hline Age & -1.04 & 0.44 & 0.02 \\
\hline Sex (male vs. female) & 15.63 & 4.42 & 0.001 \\
\hline Marijuana use (current use vs. no current use) & 0.46 & 5.93 & 0.94 \\
\hline
\end{tabular}

C. Working Memory

\begin{tabular}{lccc}
\hline Variable & Beta & SE (Beta) & P \\
\hline \% CD4+CD38+HLA-DR+ ${ }^{a}$ & 0.83 & 1.34 & 0.54 \\
sCD14 & 0.25 & 4.75 & 0.96 \\
HIV duration & -0.06 & 0.03 & $\mathbf{0 . 0 4}$ \\
Age & -0.05 & 0.47 & 0.92 \\
Sex (male vs. female) & -0.82 & 4.69 & 0.86 \\
Marijuana use (current use vs. no current use) & 6.02 & 5.91 & 0.32 \\
\hline & & $\mathrm{R}^{2}=0.18$ & \\
\hline
\end{tabular}

D. Processing Speed

\begin{tabular}{lccc}
\hline Variable & Beta & SE (Beta) & P \\
\hline \% CD4+CD38+HLA-DR+ ${ }^{a}$ & -2.81 & 1.36 & $\mathbf{0 . 0 4 7}$ \\
sCD14 & -0.75 & 4.82 & 0.88
\end{tabular}




\begin{tabular}{lccc} 
HIV duration & -0.05 & 0.03 & 0.12 \\
Age & -0.19 & 0.47 & 0.69 \\
Sex (male vs. female) & -2.71 & 4.77 & 0.57 \\
Marijuana use (current use vs. no current use) & 11.60 & 6.0 & 0.06 \\
\hline & & $\mathrm{R}^{2}=0.31$ & \\
\hline E. Full Scale IQ & & & \\
\hline Variable & Beta & SE (Beta) & P \\
\hline \% CD4+CD38+HLA-DR+ ${ }^{a}$ & -0.91 & 1.18 & 0.45 \\
sCD14 & -5.00 & 4.19 & 0.24 \\
HIV duration & -0.05 & 0.02 & $\mathbf{0 . 0 3}$ \\
Age & -0.47 & 0.41 & 0.26 \\
Sex (male vs. female) & 6.21 & 4.14 & 0.26 \\
Marijuana use (current use vs. no current use) & 2.77 & 5.21 & 0.60 \\
\hline & & \multirow{2}{*}{$\mathrm{R}^{2}=0.34$} & \\
\hline
\end{tabular}

$a_{\%}$ of CD4+ T-cells expressing CD38 and HLA-DR;

HIV, human immunodeficiency virus; SE, standard error; sCD14, soluble CD14; IQ, intelligence quotient 